hep-th/0702111

MIT-CTP-3812

\title{
Phases of Five-Dimensional Black Holes
}

\author{
Henriette Elvang ${ }^{a}$, Roberto Emparan ${ }^{b, c}$ and Pau Figueras ${ }^{c}$ \\ ${ }^{a}$ Center for Theoretical Physics \\ Massachusetts Institute of Technology, Cambridge MA 02139, USA \\ ${ }^{b}$ Institució Catalana de Recerca i Estudis Avançats (ICREA) \\ ${ }^{c}$ Departament de Física Fonamental \\ Universitat de Barcelona, Diagonal 647, E-08028 Barcelona, Spain \\ elvang@lns.mit.edu, emparan@ub.edu, pfigueras@ffn.ub.es
}

\begin{abstract}
We argue that the configurations that approach maximal entropy in five-dimensional asymptotically flat vacuum gravity, for fixed mass and angular momentum, are 'black Saturns' with a central, close to static, black hole and a very thin black ring around it. For any value of the angular momentum, the upper bound on the entropy is equal to the entropy of a static black hole of the same total mass. For fixed mass, spin and area there are families of multi-ring solutions with an arbitrarily large number of continuous parameters, so the total phase space is infinite-dimensional. Somewhat surprisingly, the phases of highest entropy are not in thermal equilibrium. Imposing thermodynamical equilibrium drastically reduces the phase space to a finite, small number of different phases.
\end{abstract}




\section{Introduction}

In this paper we study the phases of black holes in five-dimensional gravity 1 with total mass $M$ and angular momentum $J$, i.e., phases in the microcanonical ensemble. A question of particular interest is which configuration maximizes the entropy for given $M$ and $J$.

In five dimensions, in addition to the Myers-Perry (MP) black holes with horizon topology $S^{3}$ [1], there exist black rings with topology $S^{1} \times S^{2}$, which imply discrete nonuniqueness in a range of parameters [2]. But black rings turn out to introduce a much larger degeneracy through multi-black hole configurations. To understand these, recall that the $S^{1}$-radius of a black ring can be made arbitrarily large, for a given mass, by thinning the ring. So, given a sufficiently thin and long ring, we can imagine putting a MP black hole at its center. This increases slightly the centripetal pull on the ring, but the effect can be counterbalanced by increasing its rotation. These configurations, dubbed black Saturns have been explicitly constructed and analyzed recently in [3]. The black ring can actually encircle the central black hole quite closely, giving rise to effects such as rotational dragging, but here we are more interested in situations where the interactions between the ring and the central black hole are small. In principle the construction method used in [3] allows to systematically add an arbitrary number of rings, but the complication grows enormously with each new ring. Solutions with two black rings (without any central MP black hole) have been constructed in [4].

A scatter-plot sampling of the parameter space of the exact solutions in [3] showed regions of the phase diagram where black Saturns are the entropically dominating solutions. We will argue here that this happens throughout the entire phase diagram: for any values of $M>0, J>0$, the phase with highest entropy is a black Saturn. Naively, one might have thought that a configuration with multiple black holes should increase its entropy by merging them all into a single black hole. That this need not be so for a black Saturn follows from two simple observations:

1. Among all single black objects of a given mass $M$, the one with maximal entropy is the static $(J=0)$ spherical black hole.

2. A black ring of fixed mass can carry arbitrarily large spin $J$ by making its $S^{1}$-radius large enough (and its $S^{2}$-radius small enough). Conversely, there is always a thin black ring of arbitrarily small mass with any prescribed value of $J$.

\footnotetext{
${ }^{1}$ More precisely: stationary vacuum solutions, $R_{\mu \nu}=0$, which are asymptotically flat and regular on and outside the black hole horizons.
} 
So we may say that static spherical black holes are the most efficient black objects (since they use up a minimal mass) for carrying entropy, and black rings are the most efficient ones for carrying spin. So, given any values of $M>0$ and $J>0$, the total entropy will be maximized by putting virtually all the mass in a central static black hole, and having an extremely long, thin and light black ring, carrying all the angular momentum. The total entropy of this black Saturn configuration approaches asymptotically, in the limit of infinitely thin ring, the entropy of a static black hole with the same total mass. We shall argue that in fact for any given value of the total angular momentum, there exist black Saturns spanning the entire range of areas

$$
0<\mathcal{A}<\mathcal{A}_{\max }=\frac{32}{3} \sqrt{\frac{2 \pi}{3}}(G M)^{3 / 2} .
$$

Here $\mathcal{A}_{\max }$ is the area of the static black hole.

We can also infer another remarkable feature of the five-dimensional black hole phase space. Black Saturns with a single black ring exhibit two-fold continuous non-uniqueness [3]: besides the total $M$ and $J$, two other continuous parameters - say, the mass of the black ring and its dimensionless 'thickness' parameter - are needed in order to fully specify the solution. Adding $n$ more rings introduces $2 n$ more continuous parameters. Moreover, a ring can have an effect as small as desired on the total black Saturn. So for generic values of $M, J$ and $\mathcal{A} \in\left(0, \mathcal{A}_{\max }\right)$ there are black Saturns with an arbitrarily large number of rings, and therefore characterized by an arbitrarily large number of continuous parameters.

The phase space thus shows a striking infinite intricacy. However, most of these solutions, in particular the ones that maximize the total area, are not in thermal equilibrium: the temperatures and angular velocities need not be the same on disconnected components of the horizon. We find that imposing thermodynamic equilibrium drastically reduces the phase space to a finite number of families (perhaps only three), each specified by a function $M(J, \mathcal{A})$.

\section{Phasing in Saturn}

We begin by recalling the known single-black-object phases (i.e., with connected horizons), namely, MP black holes and black rings. We only consider solutions with angular momentum in a single rotation plane. In order to eliminate awkward factors from the formulas, 
we will work with rescaled spins and areas 2

$$
\tilde{J} \equiv \sqrt{\frac{27 \pi}{32 G}} J, \quad \tilde{\mathcal{A}} \equiv \sqrt{\frac{27}{256 \pi G^{3}}} \mathcal{A} .
$$

The MP black hole phase with mass $M_{h}$ and angular momentum $J_{h}$ is characterized by the area

$$
\tilde{\mathcal{A}}_{h}=2 \sqrt{2\left(M_{h}^{3}-\tilde{J}_{h}^{2}\right)},
$$

and the black rings with mass $M_{r}$, in parametric form, by area and angular momentum

$$
\tilde{\mathcal{A}}_{r}=2 \sqrt{M_{r}^{3} \nu(1-\nu)}, \quad \tilde{J}_{r}^{2}=M_{r}^{3} \frac{(1+\nu)^{3}}{8 \nu} .
$$

To characterize their sizes, we introduce the circumferential radius of the MP black hole in the rotation plane

$$
R_{h}=2 \sqrt{\frac{G}{3 \pi}} \sqrt{\frac{2 M_{h}}{1-\tilde{J}_{h}^{2} / M_{h}^{3}}}
$$

and the $S^{1}$-radius of the inner rim of the ring,

$$
R_{1}=2 \sqrt{\frac{G}{3 \pi}} \sqrt{M_{r} \frac{1-\nu}{\nu}} .
$$

The dimensionless parameter $\nu \in(0,1)$ is a 'thickness' parameter for the black ring. 3 If we fix $M_{r}$, then as $\nu \rightarrow 0$ the angular momentum and $R_{1}$ both go to infinity, and the area approaches zero: this is the limit of an infinitely thin ring of infinite radius. In the opposite limit, as $\nu \rightarrow 1$ we find a naked singularity with zero area. The same singular solution is found in the extremal limit of MP black holes, $\tilde{J}_{h}^{2} \rightarrow M_{h}^{3}$. The phase diagram with these solutions is shown in figure 1, For every value of $\tilde{J}$, there is at least one black object, and for $\sqrt{27 / 32}<\tilde{J}<1$ there are three of them.

Generically, adding spin for fixed mass reduces the area, as also happens for the fourdimensional Kerr solution. So for a given value of $M$, the maximal entropy is attained by the static black hole,

$$
\tilde{\mathcal{A}}_{\text {max }}=(2 M)^{3 / 2} \text {. }
$$

In principle any solution that is regular on and outside the horizon, even if it consists of disconnected horizons, is an allowed phase. So now we consider multi-black hole configurations. In classical General Relativity we are free to fix a scale, which we will take to be

\footnotetext{
${ }^{2}$ For solutions with a single black object of unit mass these coincide with the reduced spin and area, $j$ and $a_{H}$, introduced in [5].

${ }^{3}$ For more precision and details see 6 .
} 


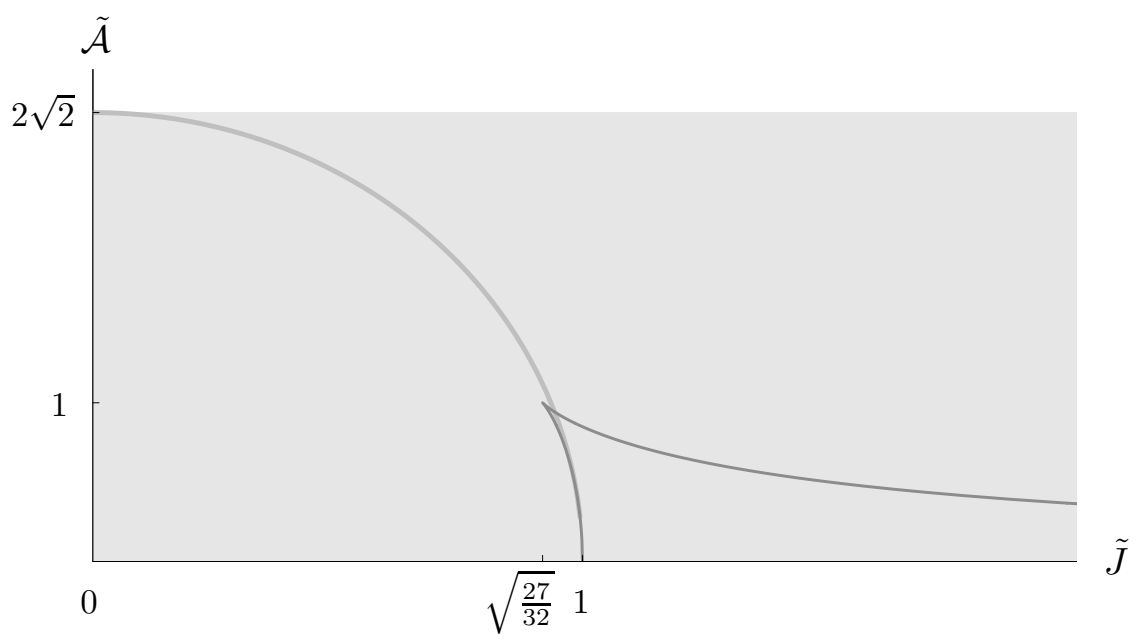

Figure 1: Phases of five-dimensional black holes including black Saturns. We fix total mass $M=1$ and plot total area vs. spin. The solid curves correspond to single MP black holes and black rings. The semi-infinite shaded strip, spanning $0 \leq \tilde{J}<\infty, 0<\tilde{\mathcal{A}}<2 \sqrt{2}$, is covered by black Saturns. Each point in the strip actually corresponds to a one-parameter family of Saturn solutions. The top end $\tilde{\mathcal{A}}=2 \sqrt{2}$ with $\tilde{J} \neq 0$ is reached only asymptotically for black Saturns with infinitely long rings. Solutions at the bottom $\tilde{\mathcal{A}}=0$ are naked singularities. For a fixed value of $\tilde{J}$ we can move from the top of the strip to the bottom by varying the spin of the central black hole $\tilde{J}_{h}$ from 0 to 1 . For fixed area we can move horizontally by having $\tilde{J}_{h}<0$ and varying the spin of the ring between $\left|\tilde{J}_{h}\right|$ and $\infty$.

the total mass $M$. So, fixing

$$
M=1
$$

we want to know which solutions exist for a given total $J$, and what their total entropy is. In particular, we want to find the configurations with maximal entropy.

The simplest black Saturns consist of a central MP black hole and a single black ring. The actual solutions are rather complicated due to the gravitational interactions between both objects, but if $R_{1} \gg R_{h}$ these effects become negligible. If $M_{h}$ is not much smaller than $M_{r}$, this requires a very thin ring, i.e., small $\nu .4$ Observe in particular that the dragging effect that the black ring has on the central black hole can be made arbitrarily small, no matter how large $\tilde{J}_{r}$, by making the ring thin and long enough. This becomes clear by recalling that black rings resemble boosted black strings, whose dragging falls off asymptotically in the transverse radial direction $r$ like $\sim R_{2} / r$, where $R_{2}$ is the radius of the $S^{2}$ of the string. So the effect near the center of the ring is at most $\sim R_{2} / R_{1} \simeq \nu$.

\footnotetext{
${ }^{4}$ Thicker rings with a tiny black hole at the center are of little interest to us here, since they give phases with entropy very close to that of single black rings.
} 
In this approximation, then, we can model a black Saturn as a simple superposition of an MP black hole and a black ring. Take

$$
\tilde{\mathcal{A}}=\tilde{\mathcal{A}}_{h}+\tilde{\mathcal{A}}_{r}, \quad M=1=M_{h}+M_{r}, \quad \tilde{J}=\tilde{J}_{h}+\tilde{J}_{r} .
$$

Comparing the plots of black Saturn phases produced using these expressions with those from the exact solutions in [3] confirms that the approximation is good when the rings are thin and long.

Having fixed the scale, configurations in this model have three free parameters, just like in the actual black Saturn solutions. So for fixed $(M, \tilde{J})=(1, \tilde{J})$, there is a two-parameter family of solutions. Let us take $\tilde{J}_{h}$ and $\nu$ as these two parameters, and ask which values of them give maximal entropy. As discussed in the introduction, we expect to achieve this by putting all the spin on a thin, large ring, of very small mass. Graphical examination of the function $\mathcal{A}\left(\tilde{J}_{h}, \nu ; \tilde{J}\right)$ for different values of $\tilde{J}$ shows indeed that the maximum area is achieved with $\tilde{J}_{h}=0$ and $\nu \rightarrow 0$. So let us set $\tilde{J}_{h}=0$, and consider small values of $\nu$. Then

$$
\left.\tilde{\mathcal{A}}\right|_{\tilde{J}_{h}=0}=2 \sqrt{2}-6 \sqrt{2} \tilde{J}^{2 / 3} \nu^{1 / 3}+O\left(\nu^{2 / 3}\right) .
$$

We indeed find that the maximal area (2.6) is reached from below as $\nu \rightarrow 0$ for any value of $\tilde{J}$.

What is then the region of the plane $(\tilde{J}, \tilde{\mathcal{A}})$ covered when we include the Saturn phases? We now argue that it is the semi-infinite strip

$$
\begin{gathered}
0<\tilde{\mathcal{A}}<\tilde{\mathcal{A}}_{\max }=2 \sqrt{2} \\
0 \leq \tilde{J}<\infty
\end{gathered}
$$

plus the point $(\tilde{J}, \tilde{\mathcal{A}})=(0,2 \sqrt{2})$ for the static black hole (of course the symmetric region with $\tilde{J}<0$ is covered by reversing the spins):

- We have seen that the upper boundary of the $\operatorname{strip}(\tilde{J}>0, \tilde{\mathcal{A}}=2 \sqrt{2})$ is approached asymptotically as the ring grows infinitely long and infinitesimally thin.

- The lower boundary $(\tilde{J} \geq 0, \tilde{\mathcal{A}}=0)$ corresponds to naked singularities. One way to reach these is to make the central black hole approach the extremal singular solution $\tilde{J}_{h}^{2} \rightarrow M_{h}^{3}$, and adjusting the spin of the ring towards $\tilde{J}-M_{h}^{3 / 2}$ while sending $M_{r} \rightarrow 0$ and $\nu \rightarrow 0$. Even if in this limit $R_{h} \rightarrow \infty$, it is easy to see that, for any finite values of the parameters, we can satisfy $R_{1} \gg R_{h}$ so our approximations hold. Thus there are regular Saturn solutions arbitrarily close to the lower boundary. 
- The solutions at the left boundary, with $(\tilde{J}=0,0<\tilde{\mathcal{A}} \leq 2 \sqrt{2})$ correspond to black Saturns where the central black hole and the black ring are counterrotating. Such configurations were studied in detail in [3]. To cover the entire range $0<\tilde{\mathcal{A}} \leq 2 \sqrt{2}$ we can simply have a central black hole rotating to get the required area, and a thin black ring counterrotating so that the angular momenta of the ring and the hole cancel. It is possible to achieve $\tilde{J}=0$ also with fatter rings.

- We can easily see that there is at least one black Saturn for any point within these boundaries. Again, the idea is to have a central black hole, in general spinning, accounting for the area $\tilde{\mathcal{A}}$, and an extremely thin and long ring carrying the required angular momentum to make up for the total $\tilde{J}$.

So we can argue, by considering only black Saturns with thin rings, that all of the strip (2.10) is covered. It is also easy to see why the scatter-plots in [3] found it hard to sample the whole strip: the solutions with the highest entropies are strongly localized in a small region of the parameter space. Focusing and increasing the size of the sample shows a clear tendency to cover the whole strip, and in particular that entropies higher than those of rotating MP black holes can occur for all $J \neq 0$.

In general there will be not just one black Saturn for every point $(\tilde{J}, \tilde{\mathcal{A}})$ in the strip, but rather a one-parameter family of them. These are guaranteed to exist by continuity, starting from the configuration with an infinitely thin ring described above, and continuously increasing $\nu$ while adequately adjusting $\tilde{J}_{h}$ to keep $\tilde{J}$ and $\tilde{\mathcal{A}}$ fixed. This gives black Saturns with fatter black rings, although at each point in the strip in general there will be restrictions on the upper value for $\nu$, and on the range of $\tilde{J}_{h}$.

When we discuss the configurations with maximal entropy, we should note that our approximation based on (2.8) actually tends to underestimate slightly the entropy of a black Saturn with a very thin ring. Roughly, the reason is that the interaction between the central black hole and the black ring is attractive, and so it decreases the system's energy. Here we are fixing the total energy, which means that the attraction leaves more rest-energy to be stored in the black hole, giving a larger entropy than in the simple approximation (2.8). We have confirmed this effect with the exact solutions in [3]. It is also possible to argue, by modeling the interaction in Newtonian terms, that this does not allow to overshoot and reach areas larger than $2 \sqrt{2}$ : for small $\nu$, the resulting increase in the area is at least $O\left(\nu^{2 / 3}\right)$ and hence smaller than the leading correction in (2.9) $)$. Other competing effects, like dragging, are even more subdominant. So our conclusion that there do exist 
black Saturns with area approaching arbitrarily close to a maximum (2.6) seems robust and holds even after including interactions.

\section{Multiple rings and further parameters}

Using the same methods we can discuss solutions with multiple black rings. Begin with two concentric black rings, and no central black hole. The exact solutions have been built in [4]. When one of the rings is much longer than the other we can use an approximation analogous to (2.8) and simply superimpose two black rings. Arguments of the kind above suggest that the maximal area will be that of a minimally spinning ring (with $\nu=1 / 2$ ), i.e., $\tilde{\mathcal{A}}=1$. In this case, a strip of unit height, extending to all $\tilde{J} \geq 0$, will be covered by these solutions. So some double-ring configurations will have higher entropy than single rings with the same mass and spin, with the maximal entropy achieved when the innermost ring has $\nu \approx 1 / 2$ and the outer ring is very thin and light.

Black Saturns with multiple rings are clearly possible too, and when the rings are thin and sparsely spaced we can model them by adding more terms to (2.8). For solutions with many rings the cumulative dragging effect may become stronger, but we can always have solutions with thinner and thinner rings where this is negligible. Each class of solutions with fixed number $n$ of rings, and therefore with $2 n$ continuous parameters, covers the entire strip (2.10).

But there is even more. So far we have restricted ourselves to solutions that rotate in a single plane. It is however possible to have both the MP black hole and the black rings rotate in the plane orthogonal to the ring plane. Black rings with two independent rotation parameters have been constructed recently [7]. For fixed mass the $S^{2}$-spin $J_{2}$ is bounded above, for reasons similar to the bound on Kerr black holes. Furthermore, it can be seen that $\left|J_{2}\right|<\left|J_{1}\right|$. But another consequence of having $J_{2} \neq 0$ is that $J_{1}$ is also bounded above, and the extremal solutions that saturate this bound, for fixed mass, have non-zero area. In other words, given $J_{1}, J_{2} \neq 0$ there is a limit on how small the mass and how large the $S^{1}$ radius of the black ring can be. But even if this second rotation puts an upper limit on the $S^{1}$-radius of a black ring, it is clear that in general it adds yet another set of continuous parameters to the phase diagram with fixed $\left(M, J_{1}=J, J_{2}=0\right)$ that we have been studying. The second spin of the central black hole, if not too large, can be cancelled

against the corresponding $S^{2}$-spin of a black ring. So with each black ring in the black Saturn we get one more continuous parameter. 
Still, multi-ring black Saturns may not exhaust the class of asymptotically flat 5D vacuum black holes. Solutions that include bubbles in combination with black holes [8, 9] may play a role. It has also been speculated that black holes with only one axial symmetry might exist [10]. All of these, if they do exist, will add further dimensions to the phase space, but we find it unlikely that they can allow for larger total area than (2.6).

It is clear that the general phase space for solutions with non-zero values of the two spins will, for the same reasons, be infinite-dimensional. It will be interesting to analyze its overall features.

\section{First law of multi-black hole mechanics}

Consider a general stationary black Saturn (or any multi-black hole solution) made of $N$ black objects labelled by $i=1 \ldots, N$. Each connected component of the horizon $H_{i}$ is generated by a Killing vector

$$
k_{(i)}=\xi+\Omega_{i} \zeta,
$$

where $\xi$ and $\zeta$ are the canonically normalized Killing vectors that generate time translations and rotations near infinity, and $\Omega_{i}$ is the angular velocity on $H_{i}$. Following standard procedure [11] we can write the ADM mass as a Komar integral on a sphere at infinity,

$$
M=-\frac{3}{32 \pi G} \int_{S_{\infty}} \epsilon_{a b c d e} \nabla^{d} \xi^{e} .
$$

Since we are in vacuum, Stokes' theorem allows us to write this as

$$
M=-\frac{3}{32 \pi G} \sum_{i} \int_{H_{i}} \epsilon_{a b c d e} \nabla^{d} \xi^{e} .
$$

Using (4.1), a standard calculation leads to the Smarr relation

$$
M=\frac{3}{2} \sum_{i}\left(\frac{\kappa_{i}}{8 \pi G} \mathcal{A}_{i}+\Omega_{i} J_{i}\right)
$$

where $\mathcal{A}_{i}$ and $\kappa_{i}$ are the area and surface gravity on $H_{i}$, and

$$
J_{i}=\frac{1}{16 \pi G} \int_{H_{i}} \epsilon_{a b c d e} \nabla^{d} \zeta^{e}
$$

is the (Komar) angular momentum of the $i$-th black object. Ref. [3] checked that (4.4) holds for the explicit solutions in [3]. We could define 'Komar masses' for each black object

$$
M_{i}=-\frac{3}{32 \pi G} \int_{H_{i}} \epsilon_{a b c d e} \nabla^{d} \xi^{e}=\frac{3}{2}\left(\frac{\kappa_{i}}{8 \pi G} \mathcal{A}_{i}+\Omega_{i} J_{i}\right),
$$


but note that the latter equalities are actually mathematical identities on each horizon, so they should not be thought of as 'true' Smarr relations.

In a similar manner, a straightforward extension of [11] yields the first law of multi-black hole mechanic: 5

$$
\delta M=\sum_{i}\left(\frac{\kappa_{i}}{8 \pi G} \delta \mathcal{A}_{i}+\Omega_{i} \delta J_{i}\right) .
$$

The phase space of black Saturn configurations with up to $N$ black objects is $2 N$-dimensional. One may use the $\left(\mathcal{A}_{i}, J_{i}\right)$ as variables in it, or perhaps better, the total $\mathcal{A}$ and $J$, and $\left(\mathcal{A}_{i}, J_{i}\right)$, $i=2, \ldots, N$. Fig. 1 is in fact a projection of the phase diagram onto the plane $(\mathcal{A}, J)$. It must be noted that, as is apparent already when $N=1$, the $\left(\mathcal{A}_{i}, J_{i}\right)$ variables do not necessarily specify a unique phase, as there may be discrete degeneracies.

\section{Thermodynamical equilibrium}

Black Saturns are valid stationary solutions, regular on and outside the horizons. But there is a glaring problem if they are to be considered as thermodynamical phases: the separate black components have in general different temperatures. Solutions with equal temperatures on all horizons are possible, but in the configurations that maximize the entropy the thin black ring has a temperature that is inversely proportional to its $S^{2}$ radius and hence diverges in the limit of zero thickness. So we find that the system can increase its entropy by creating a large temperature gradient within itself! In other words, it looks like the highest entropy state is not in thermal equilibrium, which seems at odds with basic thermodynamical principles.

A similar problem arises with the horizon angular velocity $\Omega$, which is the intensive 'potential' for the angular momentum and therefore would be expected to be homogeneous in a situation of mechanical rotational equilibrium. For a very thin and light black ring $\Omega \sim 1 / R_{1}$ is very small, so in the black Saturns with near-maximal area it is possible to arrange for $\Omega_{h}=\Omega_{r}$. Nevertheless, generic stationary black Saturns have a variety of different $\Omega_{i}$.

In order to understand better these discrepancies, recall that black hole thermodynamics (as opposed to black hole mechanics) really makes sense only when Hawking radiation is included. In the presence of radiation, a generic black Saturn cannot be in thermal equilibrium, even in the microcanonical ensemble. Even if $T$ is homogeneous, the radiation

\footnotetext{
${ }^{5} \mathrm{~A}$ first law and a Smarr relation of this kind were found to hold in [9] for explicit solutions with multiple static black holes in Kaluza-Klein cylinders.
} 


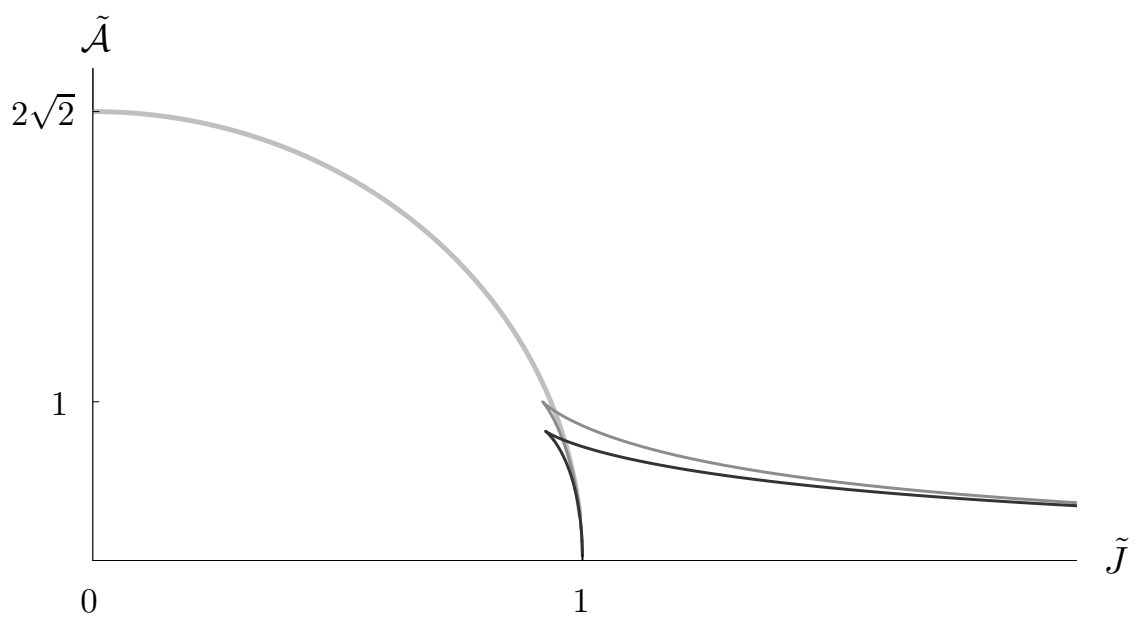

Figure 2: Phases in thermodynamical equilibrium. Besides the gray curves for MP black holes and black rings, we have the solid black curve of single-ring black Saturns for which the central black hole and the black ring have equal temperature and equal angular velocity. There is only one black Saturn (and not a continuous family of them) for each point in this curve. The minimum $\tilde{J}$ along the curve is $\approx 0.9245$, slightly above $\sqrt{27 / 32}$, and the maximum total area is $\tilde{\mathcal{A}} \approx 0.81$. Note that black Saturns are never entropically dominant among the phases in thermodynamical equilibrium. In the text we argue that it is unlikely that multi-ring black Saturns exist as states in thermodynamical equilibrium. Barring more exotic possibilities, this diagram may then contain all thermodynamical equilibrium states of 5D black holes.

cannot be in mechanical equilibrium between two black objects with different values of $\Omega$. In fact the radiation will couple the different black objects and drive the system towards thermodynamical equilibrium. In the absence of this radiation each black object acts, to some extent, as a separate thermodynamical system of its own. (A further issue comes from the fact that black Saturns within wide parameter ranges are expected to be classically dynamically unstable. We will discuss further this point in the final section.)

If we consider a black Saturn with a single ring, then requiring equal temperatures and angular velocities for the central black hole and the black ring imposes two conditions on the parameters. This removes entirely the continuous non-uniqueness, leaving at most discrete degeneracies. Black Saturns in thermodynamical equilibrium thus form a curve in the plane $(\tilde{J}, \tilde{\mathcal{A}})$. This curve, generated from the exact solutions in [3], is shown in fig. 2,

The qualitative properties of the curve are easy to understand. Consider a black Saturn with a thin (though not necessarily very thin) black ring. In order for the temperature of the central black hole and the black ring to be the same, the black hole radius must be roughly the same as the ring's $S^{2}$ radius [6]. Then, the longer the ring, the larger the 
fraction of the total mass it carries. In particular, as $\tilde{J} \rightarrow \infty$ most of the mass is in the ring, so the black Saturn curve asymptotes to that of a single black ring. As the black ring gets thicker a larger fraction of the mass goes into the central black hole, and the total entropy is only a fraction of the entropy of a single ring. When the black ring is fat, the two black objects get distorted and flattened along the rotation plane, approaching the singular zero-area limit at $\tilde{J}=1$, common to black rings and MP black holes. Although not shown in the figure, along the equilibrium curve the angular velocity is smaller and the temperature higher than those of a single ring of the same $\tilde{J}$. The reason is that in a black Saturn the angular momentum is distributed over more objects (hence $\Omega$ is lower) that are thinner (hence hotter) than in the single black ring.

It looks much more difficult to impose equality of all temperatures and all angular velocities on solutions with two or more black rings (whether with a central MP black hole or not). The reason is that fixing $T$ and $\Omega$ determines uniquely a black ring [6]. So once we have one ring, if we try to put in another one with the same $T$ and $\Omega$, we are actually trying to put in the same ring. There may be a slim chance that, due to non-linearities, a di-ring with two very close-by rings of the same $T$ and $\Omega$ exist. This can be settled by examining the exact solutions in [4]. But clearly this is a strongly constrained possibility, and it seems highly unlikely that an arbitrary number of black rings in thermodynamical equilibrium can be piled on top of each other.

So the condition of thermodynamical equilibrium not only removes all continuous nonuniqueness, but it also reduces to a handful the families of possible black objects: MP black holes, black rings, single-ring black Saturns — and this may be all. Barring the possibility of multi-ring black Saturns, and of the conjectural solutions mentioned at the end of section 3 , all the black hole phases in thermodynamical equilibrium would be present in figure 2. Up to a few degeneracies, these phases are specified by functions $M(J, \mathcal{A})$. So the phase space in thermodynamic equilibrium is two-dimensional, and shows only discrete non-uniqueness.

Finally, note that when the second angular momentum is turned on, the phase space of thermodynamic equilibrium becomes three-dimensional, with energy function $M\left(J_{1}, J_{2}, \mathcal{A}\right)$. It seems likely that this phase space consists only of extensions of the families of solutions considered above. 


\section{Discussion}

For upon immediately directing my telescope at Saturn, I found that things there had quite a different appearance from that which they had previously been thought by most men to have.

Christiaan Huygens, Systema Saturnium (1659)

The phase structure we have uncovered has certainly quite a different appearance from that which it had previously been thought to have.

An infinite-dimensional phase space, due to configurations with an arbitrarily large number of black holes, is also known to occur in the context of black holes localized in Kaluza-Klein cylinders, or 'caged' black holes [12]. However, configurations with multiple caged black holes are all expected to increase their entropy by merging into a single black hole. What is most remarkable about black Saturns is not so much that they are possible, but that they maximize the entropy. The essential idea behind this maximization consists of transferring all the spin of the black hole (which tends to reduce the entropy) into orbital angular momentum carried by a very light object at large radius. In general this can be achieved through non-stationary configurations with, say, faraway gravitational waves, or small black holes, carrying the angular momentum, plus a central black hole close to maximal area. This possibility was in fact considered in [13], but black Saturns realize it solely in terms of stationary solutions.

Black Saturns with very thin and long rings are loosely bound configurations, with negligible binding energy. But more importantly, they are expected to be dynamically unstable. Very thin black rings are likely to suffer from Gregory-Laflamme instabilities [14, 2, 15, 6, 16]. If, as a result, the black ring fragments into smaller MP-like black holes, these will fly apart. This final configuration is reminiscent of those considered in [13] for the decay of ultra-spinning MP black holes in $D \geq 6$. There, the possibility of increasing the area through fragmentation was one of several telltale signs of a classical ultraspinning instability. We have found that five-dimensional rotating MP black holes and black rings might similarly increase their entropy through fragmentation into a black Saturn (which may then fragment further). Could this signal a classical instability of all five-dimensional black holes with $J \neq 0$ ?

Before jumping to this conclusion, it is important to bear in mind that the existence of a configuration with higher total area is merely suggestive of a possible dynamical instability. In addition, there must exist some plausible classical dynamical evolution that drives the 
initial configuration towards another one close enough to the putative final state (through a curvature singularity, if fragmentation is to occur). In this respect, it seems difficult for an MP black hole, or a black ring, to dynamically evolve into a black Saturn of near-maximal area, which has a black ring of much larger extent on the rotation plane than the initial black hole. But it could possibly evolve towards a black Saturn of roughly the same size as the initial black hole, if the final area were higher. This remains to be investigated.

While it may seem striking that the configuration of maximal entropy is classically unstable (except at $J=0$ : the static black hole is stable [17]), it should be recalled that it is not in thermal equilibrium, so usual thermodynamic reasoning does not really apply here. When Hawking radiation is taken into account, the very thin black ring of black Saturn (or, if it fragments, the very small black holes flying away) will evaporate much faster than the central black hole that carries most of the mass. An important problem is to find the dynamically stable configurations with maximal entropy among those that are in stable thermodynamical equilibrium with Hawking radiation in the microcanonical ensemble. It seems possible that this consists of an MP black hole surrounded by spinning radiation. However, the thermodynamics of other ensembles is likely to be quite different [6]. Another question to bear in mind is that black Saturns with maximal area require infinite volume. In a finite volume with fixed energy, maximal area may not always be attained by a black Saturn. This will be the case when the volume is large compared to, say, the Schwarzschild radius for that energy. But if the box could barely fit a single MP black hole, or a single black ring, then it might not fit a black Saturn of larger area. All these issues must be sorted out depending on the specific application of these ideas one is considering.

Dynamically stable black Saturns having only $M$ and $J$ as conserved charges are likely to exist at least in Einstein-Maxwell theories. If the black ring in the configuration carries a dipole charge [5] it can saturate an extremal (not BPS) bound, in which case the GregoryLaflamme instability is expected to disappear. Thin dipole black rings have also been argued to be radially stable [6]. So dipole black Saturns can presumably be made classically stable. Supersymmetric multi-ring black Saturns, also expected stable, have been described in [18]. It has been observed that in some cases they can have larger entropy than a single black hole with the same conserved charges. Semi-qualitative arguments similar to the ones in this paper might help understand this effect.

Thin black rings have been argued to exist in any $D \geq 5$, achieving equilibrium in essentially the same manner as in $D=5$ [15, 6]. So black Saturns should exist, too, in any $D \geq 5$ and in particular the ones that maximize the entropy should share the same 
features that we have described in this paper. A semi-infinite strip in the $(\tilde{J}, \tilde{\mathcal{A}})$ plane will then be covered by an infinite number of families of solutions, but only a few of them are expected to be in thermal equilibrium.

Finally, our discussion of the phase space has been wholly classical. In quantum gravity the continuous parameters will become discrete. Furthermore, there will be a lower, Plancklength bound on the size of the $S^{2}$ of thin black rings in a black Saturn. This will limit the thinness of the ring and force the entropy of a black Saturn to be finitely below the maximal value.

\section{Acknowledgements}

We thank Gary Horowitz for comments on the first version of this paper. HE was supported by a Pappalardo Fellowship in Physics at MIT and by the US Department of Energy through cooperative research agreement DE-FG0205ER41360 . RE and PF were supported in part by DURSI 2005 SGR 00082, CICYT FPA 2004-04582-C02-02 and EC FP6 program MRTN-CT-2004-005104. PF was also supported by an FI scholarship from Generalitat de Catalunya.

\section{References}

[1] R. C. Myers and M. J. Perry, "Black Holes In Higher Dimensional Space-Times," Annals Phys. 172, 304 (1986).

[2] R. Emparan and H. S. Reall, "A rotating black ring in five dimensions," Phys. Rev. Lett. 88 (2002) 101101 |arXiv:hep-th/0110260]; for a review, see "Black rings," Class. Quant. Grav. 23 (2006) R169 arXiv:hep-th/0608012].

[3] H. Elvang and P. Figueras, "Black Saturn," arXiv:hep-th/0701035.

[4] H. Iguchi and T. Mishima, "Black di-ring and infinite nonuniqueness," arXiv:hep-th/0701043.

[5] R. Emparan, "Rotating circular strings, and infinite non-uniqueness of black rings," JHEP 0403 (2004) 064 arXiv:hep-th/0402149.

[6] H. Elvang, R. Emparan and A. Virmani, "Dynamics and stability of black rings," JHEP 0612 (2006) 074 arXiv:hep-th/0608076. 
[7] A. A. Pomeransky and R. A. Sen'kov, "Black ring with two angular momenta," arXiv:hep-th/0612005.

[8] R. Emparan and H. S. Reall, "Generalized Weyl solutions," Phys. Rev. D 65 (2002) 084025 arXiv:hep-th/0110258.

[9] H. Elvang, T. Harmark and N. A. Obers, "Sequences of bubbles and holes: New phases of Kaluza-Klein black holes," JHEP 0501 (2005) 003 arXiv:hep-th/0407050.

[10] H. S. Reall, "Higher dimensional black holes and supersymmetry," Phys. Rev. D 68 (2003) 024024 [Erratum-ibid. D 70 (2004) 089902] [arXiv:hep-th/0211290].

[11] J. M. Bardeen, B. Carter and S. W. Hawking, "The Four laws of black hole mechanics," Commun. Math. Phys. 31 (1973) 161.

[12] For reviews, see: B. Kol, "The phase transition between caged black holes and black strings: A review," Phys. Rept. 422 (2006) 119 arXiv:hep-th/0411240]; T. Harmark and N. A. Obers, "Phases of Kaluza-Klein black holes: A brief review," arXiv:hep-th/0503020.

[13] R. Emparan and R. C. Myers, "Instability of ultra-spinning black holes," JHEP 0309 (2003) 025 arXiv:hep-th/0308056.

[14] R. Gregory and R. Laflamme, "Black strings and p-branes are unstable," Phys. Rev. Lett. 70, 2837 (1993) arXiv:hep-th/9301052.

[15] J. L. Hovdebo and R. C. Myers, "Black rings, boosted strings and Gregory-Laflamme," Phys. Rev. D 73, 084013 (2006) arXiv:hep-th/0601079].

[16] For a review on instabilities of higher-dimensional black objects, see T. Harmark, V. Niarchos and N. A. Obers, "Instabilities of black strings and branes," arXiv:hep-th/0701022.

[17] A. Ishibashi and H. Kodama, "Stability of higher-dimensional Schwarzschild black holes," Prog. Theor. Phys. 110 (2003) 901 arXiv:hep-th/0305185.

[18] J. P. Gauntlett and J. B. Gutowski, "Concentric black rings," Phys. Rev. D 71, 025013 (2005) arXiv:hep-th/0408010; "General concentric black rings," Phys. Rev. D 71 (2005) 045002 arXiv:hep-th/0408122]. 